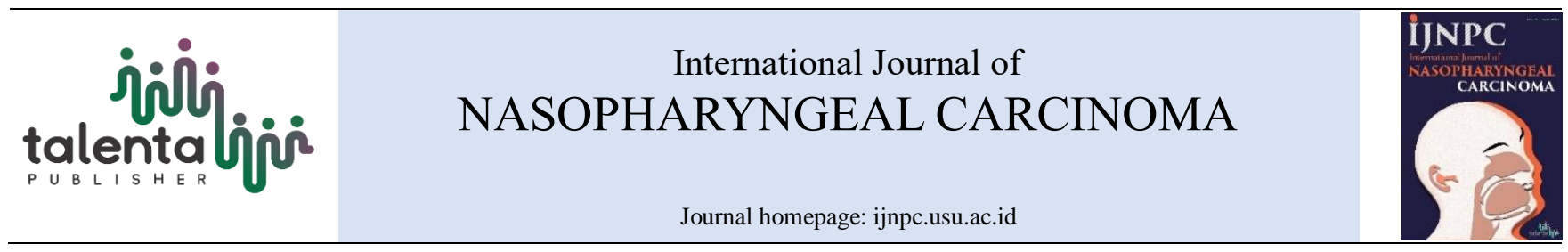

\title{
RECURRENT NASOPHARYNGEAL CARCINOMA: PROBLEM AND MANAGEMENT
}

\author{
Rusina Hayati* \\ Departement of Otorhinolaryngology Head Neck Surgery, Ulin General Hospital Banjarmasin
}

\begin{abstract}
Introduction: Treatment results for nasopharyngeal carcinoma (NPC) have been improved, but there were still some patients who developed localized persistent or local recurrent. Local recurrence still represents a major cause of mortality and morbidity also management still challenging issues.

Case Report: A 27-year-old woman presented with bilateral conductive hearing loss, dysphagia, rhinorrhoea, epistaxis, and cranial nerve palsy after chemo radiation for NPC. The results of nasopharyngoscopy, CT scan, and PET scan were not found a mass in the nasopharynx. The result of nasopharyngeal biopsy result was undifferentiated carcinoma. Patient diagnosed with recurrent NPC and planned for re-radiotherapy.

Conclusion: After complete treatment for NPC we must immediately ensure recurrence and choose the next appropriate treatment.
\end{abstract}

\section{Article Info}

Keywords:

Recurrent nasopharyngeal carcinoma, chemo radiation, undifferentiated carcinoma

\section{*Corresponding author: \\ Address: Jl. A. Yani No.79, Sungai Baru, Kec. Banjarmasin Tengah, Kota Banjarmasin, Kalimantan Selatan 70233}

e-mail: rusinahayati09@gmail.com

\section{CASE REPORT}

Treatment result for nasopharyngeal carcinoma (NPC) have improved due to the advances in imaging, radiotherapy techniques, and the use of combined treatment with chemotherapy. However there are patients who developed localized persistent or recurrent carcinoma after completion of combined therapy. Local recurrence still represents a major cause of mortality and morbidity in advanced stages and management of local failure remains a challenging issue in NPC.

A 27-year-old female patient, a midwife, came to ENT outpatient ward at Ulin hospital on November 18th 2018 with chief complaint otorrhoea from the left ear for 6 months, which was getting worse. The discharge can be runny or thick, but no smell. Bilateral hearing loss and tinnitus for 5 years, more severe on the left ear.

She has been feeling stuck and tastes a lot of phlegm in the throat every day when waking up in the morning for 3 months but improved after drinking. There was a bilateral rhinorrhoea for 9 years, the discharge was runny nose until thick snot. The patient got epistaxis 4 days ago and there was a history of epistaxis 9 years ago. Often sneezing for 4 years. No complaint of a lump in the neck. History of double vision 5 years ago. There was head feeling heavy for 5 years, especially when looking down.

She has been diagnosed as NPC 5 years ago (August 22nd 2013) according to nasopharyngeal biopsy result was undifferentiated carcinoma nasopharynx (picture 1). The patient has been received radiotherapy 25 times at Ulin Hospital Banjarmasin and 13 times at Cipto Mangunkusumo Hospital Jakarta. Chemotherapy was given 6 times, 3 times each at Cipto Mangunkusumo Hospital Jakarta and Ulin Hospital Banjarmasin performed by a hemato-oncology specialist.
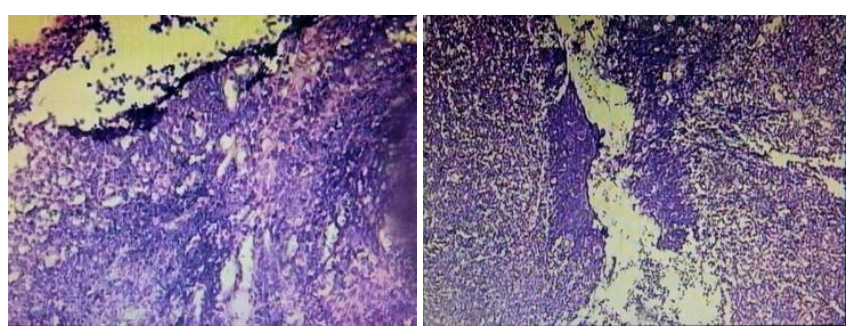

Picture 1. The nasopharynx biopsy result at August $22^{\text {nd }} 2013$

Head CT scan result after chemoradiation on February 2nd, 2014 was normal nasopharynx space and abdominal USG on February 10th, 2014 there were no abnormalities. Whole-body PET scan August 28th, 2014 there was no mass at the nasopharynx, radioopaque at the left ethmoidal and sphenoidal sinus, no lymphadenopathy with FDG catch in the mediastinum, hilus, and bilateral axilla. Audiometry at February 13th 2014, the result was bilateral conductive hearing loss There was a history of often eat salted fish, but no history of smoking and drink alcoholic beverages. There was no family history has a malignant tumor.

On August 8th, 2017 she has been diagnosed bilateral effusion otitis media. The patient underwent a bilateral ventilating tube insertion and nasopharyngeal biopsy under general anesthesia on August 16th, 2017. The result of nasopharyngeal biopsy (August 26th 2017) was undifferentiated carcinoma (WHO type 3) (Picture 2).

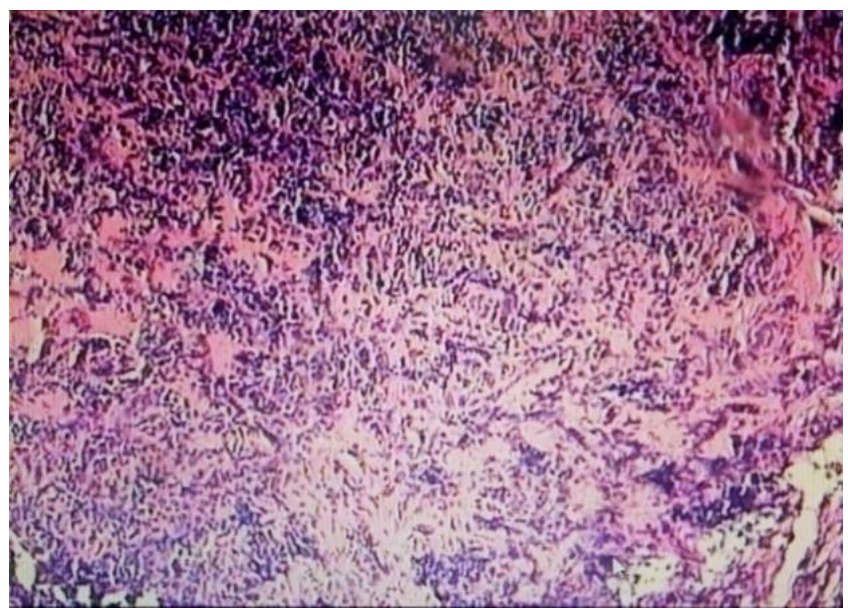

Picture 2. The nasopharynx biopsy result at August $26^{\text {th }} 2017$

On physical examination we obtained good general condition, awareness was conscious, vital sign within normal limit. There was no anemia/jaundice/dyspnoea/cyanosis. The patient can be delivered well.

On the right ear there was no discharge and intact tympanic membrane, but retraction, no conus of light. On the left ear there was serous discharge, central perforation at the tympanic membrane. From anterior rhinoscopy, there was no discharge, normal size/color of the concha, no mass, and palate 
molle phenomenon positive on both sides. On the throat there was a deviation of the tongue and negative gag reflex. There was no neck mass.

Hematology laboratory result obtained Haemoglobin 10,7 g/dl, Leukocyte count 4,8x103, trombocyte $353 \times 103$, Haemotocrit $33 \%$, Ureum $9 \mathrm{mg} / \mathrm{dl}$ and Creatinin $0,7 \mathrm{mg} / \mathrm{dl}$.

Nasopharyngoscopy on November 17th, 2018 there was thick and purulent discharge, some were attached dry secretion, clear like glass. There was not look like a mass on the Rossenmuller fossa, roof, and wall of the nasopharynx, although some were difficult to evaluate because they were closed secretion (Picture 3 ).

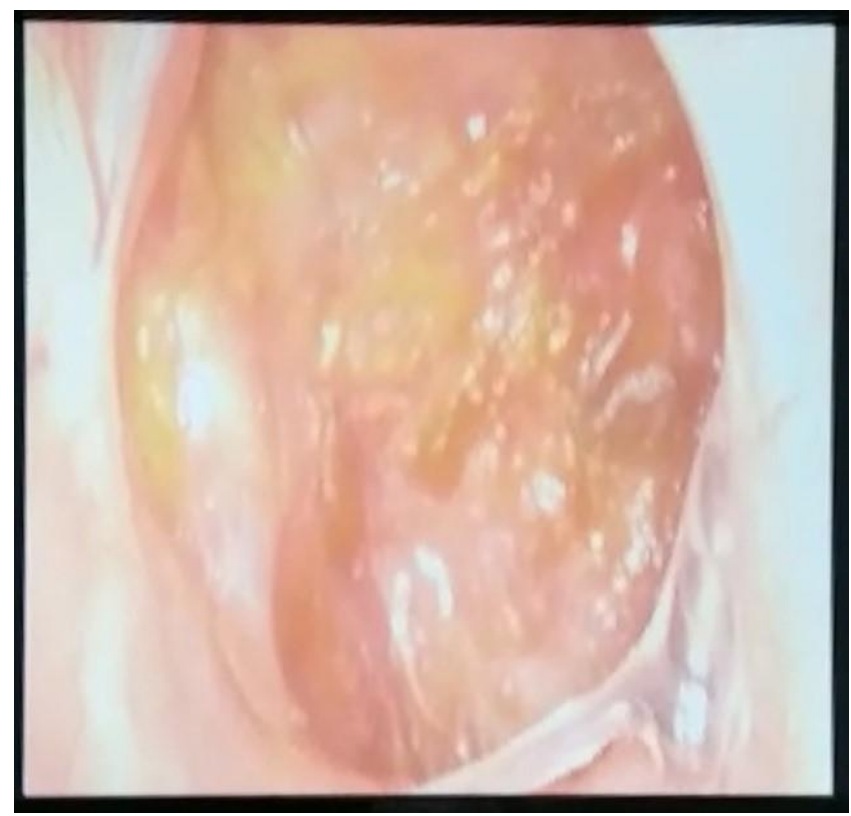

Picture 3. Nasopharyngoscopy does not appear mass, just thick secretion

Currently a diagnosis was made as recurrent NPC with the differential diagnosis was persistent NPC and bilateral serous otitis media. On the head and neck CT scan on November 22nd 2018 we obtained a conclusion as NPC residual in the ethmoid sinus, not very contrasting, differential diagnosis of fibrosis (Picture $4 \& 5$ )

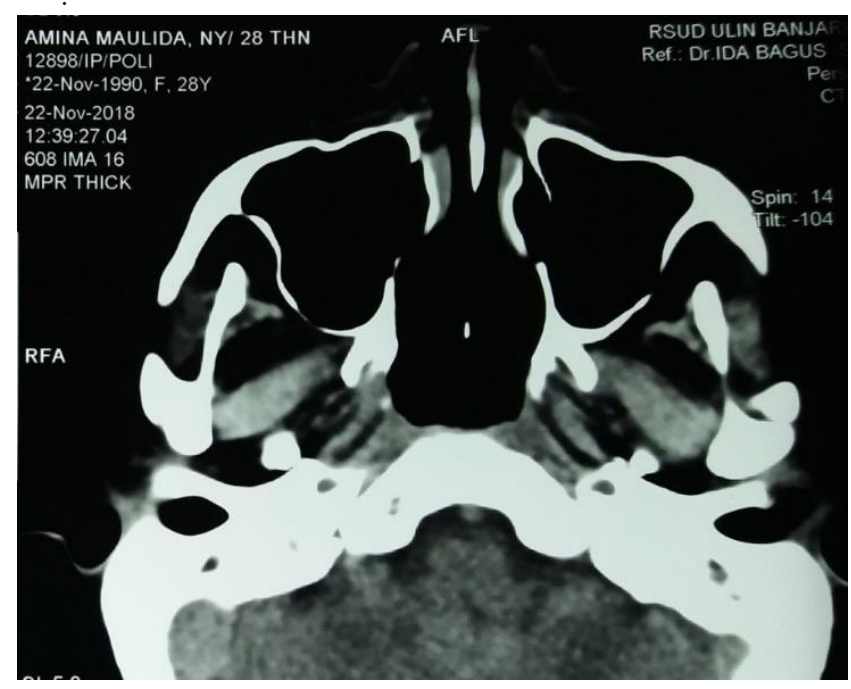

Picture 4. The nasopharyng does not appear mass

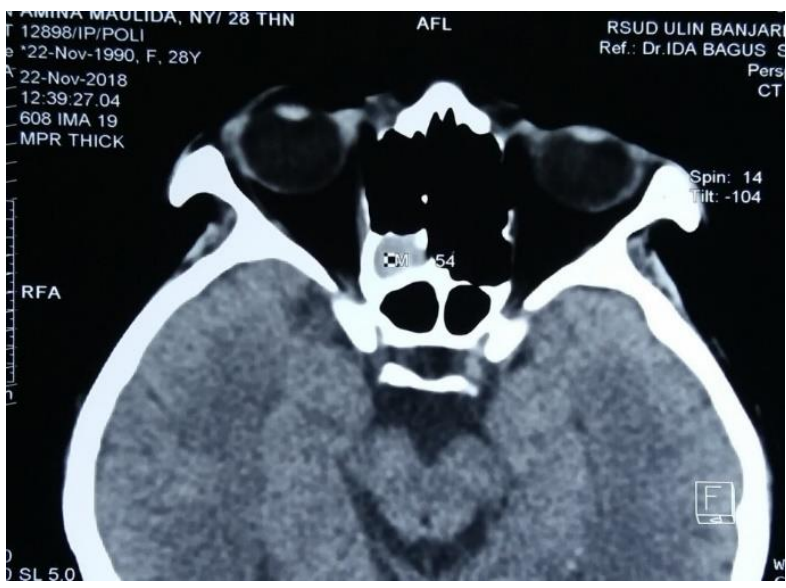

Picture 5. The right ethmoidal sinus is radiopaque

\section{DISCUSSION}

Nasopharyngeal carcinoma (NPC) is a rare malignancy in most of the world but common in Southern China. The incidence and mortality in China and the constituents area are 3.16 and 1.53 per 100,000 people, respectively, according to the national population in 2010 . The world age-standardized incidence and mortality were 2.44 and 1.18 per 100,000 people, respectively [1]. Overall incidence NPC in Indonesia 5.66/100,000, equaling roughly 1000 new cases per month, reflected a major health problem in Indonesia particularly because most of these patients were referred to the hospital a late-stage [2].

Different factors are thought to contribute to the pathogenesis of cases in endemic and non-endemic regions. Epstein-Barr virus (EBV) infection, environmental factors, and genetic predisposition are proposed to be the main contributing factors in endemic regions, while the classic risk factors for other head and neck tumors such as smoking, alcohol and human papillomavirus (HPV) infection are thought to account more for cases in non-endemic areas [3]

Recurrent NPC is defined as tumor relapse after the achievement of complete remission with radical chemotherapy. Recurrent NPC can further be subdivided into local and regional recurrence [4]. Local recurrence of NPC become less common since the use of intensity-modulated radiotherapy and chemotherapy [5]. Despite significant improvements in local control due to advances in radiotherapy, local recurrence, and residual disease remain the main reasons for failure in patients with advances nasopharyngeal carcinoma. Local alone and regional alone failures respectively account for $70 \%$ and $25 \%$ of rnpc cases, and $8 \%-28 \%$ of patient experience synchronous locoregional failure [4]. The outcome for patients with recurrent or metastatic NPC is very poor, with median overall survival (OS) about 20 months [6].

Domestic and foreign studies have indicated that the clinical features of recurrent NPC may be different from primary NPC. The incidence of recurrent NPC was higher in men than in women. The male to female ratio was about 2.5 to 1 in some report and the patient median age is 46 years. The mainly pathologic type of newly diagnosed patients in regions with high incidence was WHO III. The usual condition was the patients with tumor over deep submucosa, basilar skull, or an intracranial area adjacent to the critical structures that might have technical difficulty or high morbidity probability for biopsy [5].

After the first course of radiotherapy, patients often present with fibrous hyperplasia of the nasopharynx and poor local circulation due to local vascular occlusion. This condition can lead to low efficacy of the treatment and many side effects from re-irradiation alone. Also, the residual tumor after the first treatment may acquire mutation or some mechanism that are likely to persist and render the tumor resistant to the treatment [7].

In this case there was a woman 27 years old after 38 times radiotherapy and 6 times chemotherapy. Evaluation with head and neck CT scan even whole-body PET scan there was no mass on the nasopharynx space, but there were still complaints of hearing loss with bilateral conduction hearing loss audiometry result. Besides that patients also complain of dysphagia, rhinorrhoea, and epistaxis. 
NPC cancer cells can easily disseminate locally in many ways into the nearby critical structure. Thus, the local spread of the tumor is usually observed as direct extension anteriorly to the nasal cavity and orbit; superiorly and posteriorly to the base of the skull, sphenoid sinus, and clivus; inferiorly to the oropharynx and/or laterally into the lateral parapharyngeal spaces. The Eustachian tube is located beside the nasopharynx and it connects the lateral wall of the nasopharynx to the middle ear [8].

NPC most commonly as a cervical lymph node enlargement; the tumor may not be clinically apparent at the time of presentation. Other presenting features of NPC include a bloody nasal discharge, or less frequently, cranial nerve palsies. Patient with NPC may also have otologic symptoms, including deafness due to the middle ear effusion, and conductive or sensorineural hearing loss after radiotherapy [9].

Early detection of persistent or recurrent disease is of paramount importance. For patients with elevated anti-EBV titers or suspicious endoscopic examination, a biopsy of the nasopharynx is then indicated to detect the recurrence in its early stages. CT scan delineates the extent of the tumor in the nasopharynx and whether it has affected the surrounding bony structure. MRI, aside from providing better images of soft tissue, also shows tumor size and extension to the skull base and intracranial cavity. The detection of the cervical lymph node metastases on presentation has improved with MRI and positron emission tomography (PET) and can be cytologically confirmed through fine needle aspiration cytology [10].

The majority of recurrences occurred within the first five years. Thus, if patient have no recurrence within five years, they are much less likely to have a recurrence at all. The prognosis after relapse varied with nodal status, stage of the first diagnosis as well as the remission duration after treatment. Local recurrences had a better prognosis than distant recurrences, while distant metastases to the bone had a similar prognosis with those to liver and multi-organ involvement [11].

In this case patient were not examined for nasopharyngeal biopsy immediately after chemoradiation. Nasopharyngeal biopsy was only performed when the procedure for grommet insertion after the diagnosis serous otitis media was confirmed.There was no difference in histopathological type between before chemotherapy and 3 years after chemotherapy.

With improvements in radiotherapy and the continous intensive awareness of comprehensive treatment, the 5-year overall survival rate is between $59.0 \%$ and $761 \%$. However, local and regional recurrence is still the main treatment failure of NPC, and the 5-year cumulative rate of nasopharynx and cervical lymph node recurrence is $15.0-22.0 \%$ [12].

Treatment of local failure is challenging, due to anatomical difficult accessibility of the nasopharynx and high risk on therapy related complication. Treatment option include surgery, reirradiation by external beam, brachytherapy or stereotactic radiotherapy, photodynamic therapy and chemotherapy. For early stage residual disease (rT1-2), a significant benefit is found when salvage treatment is performed. Radiation and surgical modalitites have comparable response, survival and complication rates. For more advanced tumors (rT3-4), the benevit salvage treatment is less clear, probably due to the high treatment related morbidity and mortality, caused by large tumor volumes and involvement of surrounding structures [13]. An accepted approach in the management of recurrent NPC is the delivery of as second round of radiotherapy for patients not eligible for nasopharyngectomy. Reradiotherapy is an effective and safe salvage treatment strategy for recurrent NPC [14].

In this case from the last head and neck CT scan result there are still doubt from the radiologist. Although the are not too different from the previous examination. It makes us difficult to make correct decision of treatment or other examination. But after planning for nasopharyngeal biopsy we must decide choice treatment to that patient.

Despite the varying success of surgery or reirradiation in salvage therapy or highly selected patients with local recurrence, a vast majority of case recurrent disease are only amenable for palliative chemotherapy [15] Recurrent NPC represent a substantial public health issue in the endemic areas of Asia. Despite technological improvements in the management of this disease entity in primary setting, the likelihood of local recurrent is significantly high [16].

\section{CONCLUSION}

One case of recurrent nasopharyngeal carcinoma has been reported, manisfesting as bilateral conductive hearing loss, dysphagia, rhinoroe, epistaxis and cranial nerve palsy. Nasopharyngoscopy, CT and PET scan there was no mass. Biopsy result was undifferentiated carcinoma nasopharynx. Early detection of persistent or recurrent disease is paramount importance. Treatment of local failure is challenging, due to anatomical difficult accessibility of the nasopharynx and high risk on therapy related complication. So we must immediately ensure recurrence and choose the next appropriate treatment for that case.

\section{REFERENCE}

[1] Zhou H, Shen G, Zhang W, Cai H, Zhou Y, Li L. 18F-FDG PET/CT for the diagnosis of residual or recurrent nasopharyngeal carcinoma after radiotherapy: a metaanalysis. Journal of Nuclear Medicine. 2016;57(3):342-7.

[2] Adham M, Kurniawan AN, Muhtadi AI, Roezin A, Hermani B, Gondhowiardjo S, et al. Nasopharyngeal carcinoma in Indonesia: epidemiology, incidence, signs, and symptoms at presentation. Chinese journal of cancer. 2012;31(4):185.

[3] Prawira A, Oosting S, Chen T, Delos Santos K, Saluja R, Wang L, et al. Systemic therapies for recurrent or metastatic nasopharyngeal carcinoma: a systematic review. British journal of cancer. 2017;117(12):1743-52.

[4] Xu T, Tang J, Gu M, Liu L, Wei W, Yang H. Recurrent nasopharyngeal carcinoma: a clinical dilemma and challenge. Current oncology. 2013;20(5):e406.

[5] Chong H, Lo P, Ngan R. Endoscopic nasopharyngectomy for recurrent nasopharyngeal carcinoma. Hong Kong J Radi-ol. 2015;18(1):37-40.

[6] Perri F, Scarpati GDV, Caponigro F, Ionna F, Longo F, Buonopane S, et al. Management of recurrent nasopharyngeal carcinoma: current perspectives. OncoTargets and therapy. 2019;12:1583.

[7] Li J-X, Lu T-X, Huang Y, Han F. Clinical characteristics of recurrent nasopharyngeal carcinoma in high-incidence area. The Scientific World Journal. 2012;2012.

[8] Weng J, Wei J, Si J, Qin Y, Li M, Liu F, et al. Clinical outcomes of residual or recurrent nasopharyngeal carcinoma treated with endoscopic nasopharyngectomy plus chemoradiotherapy or with chemoradiotherapy alone: a retrospective study. PeerJ. 2017;5:e3912.

[9] KEFELİ AÜ, ATASOY BM, ÖZGEN Z, Murat S, Fuat D, Faysal D. Recurrence of nasopharyngeal carcinoma in the mastoid region: Therapeutic approach of a rare clinical entity. Marmara Medical Journal. 2014;27(2):129-31.

[10] Korkut AY, Teker AM, Kahya V, Gedikli O, Somay A. Middle ear recurrence in nasopharyngeal carcinoma: a case report. The Journal of International Advanced Otology. 2011;7(2):268.

[11] Suárez C, Rodrigo JP, Rinaldo A, Langendijk JA, Shaha AR, Ferlito A. Current treatment options for recurrent nasopharyngeal cancer. European Archives of Oto-Rhino-Laryngology. 2010;267(12):1811-24.

[12] Peng X, Chen S, Du C, Yang P, Liang S, Zhang G, et al. Clinical features and prognostic factors in patients with nasopharyngeal carcinoma relapse after primary treatment. Head Neck Oncol. 2013;5(2):21.

[13] Li J, Lu T, Huang Y, Han F, Chen C, Xiao W. Clinical features of 337 patients with recurrent nasopharyngeal carcinoma. Chin J Cancer. 2010;29(1):82-6.

[14] Agas RAF, Yu KKL, Sogono PG, Co LBA, Jacinto JKM, Bacorro WR, et al. Reirradiation for recurrent nasopharyngeal carcinomas: experience from an academic tertiary center in a low-to middle-income country. Journal of global oncology. 2019;5:1-14.

[15] Stoker S, van Diessen J, De Boer J, Karakullukcu B, Leemans C, Tan I. Current treatment options for local residual nasopharyngeal carcinoma. Current treatment options in oncology. 2013;14(4):475-91.

[16] Lee A, Ma B, Ng WT, Chan A. Management of nasopharyngeal carcinoma: current practice and future perspective. J Clin Oncol. 2015;33(29):3356-64. 